\title{
Diversidad florística y cambios en las coberturas de la cuenca del humedal Jaboque y el parque La Florida (Bogotá, Colombia)
}

\author{
Diego Mauricio Cabrera-Amaya*, Catalina Lopera-Doncel, Maribel Yesenia Vásquez-Valderrama, \\ Mónica Sandoval-Ramos, José Wilfredo López-Cruz \\ Jardín Botánico de Bogotá José Celestino Mutis
}

\begin{abstract}
Resumen
El humedal Jaboque se ubica en medio de la ciudad de Bogotá y es uno de los remanentes de mayor tamaño de la red de humedales y lagos que existió en la Sabana de Bogotá. En este estudio se hizo la caracterización florística de la vegetación en este humedal y en el parque La Florida y se determinaron los cambios en las coberturas vegetales ocurridos entre el 2004 y el 2016. Se encontraron 306 especies, 207 géneros y 91 familias. Las familias con mayor riqueza fueron Asteraceae, Cyperaceae, Myrtaceae, Solanaceae y Fabaceae. Con respecto a las coberturas vegetales evaluadas, se encontró que las más abundantes fueron el juncal Schoenoplectus californicus y el camalotal Bidens laevis. Este último fue el que más cambió, pues su área tuvo un aumento de 73,2 \%. Las especies encontradas y los cambios de vegetación observados evidenciaron un leve mejoramiento en la transformación y la degradación ambiental provocados por la urbanización, la sedimentación y la contaminación de los cuerpos de agua y de las zonas terrestres en el área de estudio. (C) 2017. Acad. Colomb. Cienc. Ex. Fis. Nat.
\end{abstract}

Palabras clave: Cobertura de la tierra; Degradación ambiental; Humedales; Sabana de Bogotá.

Floristic diversity and changes in land cover of the Jaboque wetland basin and La Florida Park (Bogotá, Colombia)

\begin{abstract}
The Jaboque wetland is located inside the urban area of Bogotá and it represents one of the largest wetlands left in the Plateau of Bogotá. In this study we made the floristic characterization of the vegetation in this wetland and in La Florida Park. We determined the changes in the vegetation cover occurred between 2004 and 2016. We found 306 species, 207 genera and 91 families. The Asteraceae, Cyperaceae, Myrtaceae, Solanaceae and Fabaceae families were those with more species. The most abundant vegetation covers in 2016 were dominated by Schoenoplectus californicus and Bidens laevis, and this last species registered the most important change, as its area increased by $73,2 \%$. These findings during the period under study evidenced a slight improvement in the transformation and environmental degradation caused by urbanization, sedimentation, and contamination of water bodies and terrestrial zones in the study area. (c) 2017. Acad. Colomb. Cienc. Ex. Fis. Nat.
\end{abstract}

Key words: Conservation; Environmental degradation; Land cover; Sabana de Bogotá; Wetlands.

\section{Introducción}

El humedal Jaboque es un ecosistema urbano de la ciudad de Bogotá que constituye uno de los relictos más grandes de humedal que sobreviven a los procesos de transformación agropecuaria y de urbanización en la Sabana de Bogotá (Schmidt-Mumm, 1998; Hernández \& Rangel, 2009). El lago del parque La Florida, contiguo al río Bogotá en la orilla opuesta a Jaboque, también hace parte de estos humedales. Actualmente quedan quince humedales urbanos reconocidos, los cuales son importantes para la capital colombiana puesto que cumplen importantes funciones como la conservación de la biodiversidad, el control de inundaciones, el control de la erosión, la retención de nutrientes, y la recarga de acuíferos de la sabana, además de constituir zonas de recreación y educación ambiental (Van der Hammen, et al., 2008). En el 2010 estos humedales ocupaban un área que apenas representaba el 1,35 \% de la extensión ocupada por lagos y humedales a principios del siglo XX en Bogotá (Escobar, 2016). Los que han sobrevivido hoy se encuentran aislados, han perdido su conectividad ecológica y presentan problemas de degradación y contaminación ambiental con graves consecuencias para su funcionamiento y su conservación. En este contexto, se hace imperativo el estudio de lugares como el humedal Jaboque y el parque La Florida, con el fin de comprender cómo se conforman, cómo funcionan y cómo son

\footnotetext{
*Correspondencia:

Diego Mauricio Cabrera-Amaya, dmcabreraa@unal.edu.co

Recibido: 01 de julio de 2017

Aceptado: 18 de septiembre de 2017
} 
sus dinámicas, para así establecer acciones de conservación y evitar que se sigan fragmentando y perdiendo su capacidad de prestar servicios ecosistémicos.

Existen algunos referentes sobre la vegetación acuática y terrestre en el humedal Jaboque y el parque La Florida, entre los cuales se destacan los estudios de la flora acuática de la Sabana de Bogotá y el valle del río Ubaté (SchmidtMumm, 1998), y los trabajos de caracterización, manejo y restauración de la vegetación del humedal Jaboque (ADESSA \& EAAB, 2006; Córdoba, 2016; Hernández \& Rangel, 2009; Montenegro, Parra, Mendivelso, Vargas, 2006). Asimismo, están los diversos estudios paleoecológicos e históricos sobre la vegetación de la Sabana de Bogotá durante el Pleistoceno (Cleef \& Hooghiemstra, 1984; Van der Hammen, 1986), los cuales pueden proporcionar pistas para entender el tipo de vegetación original de los humedales de la región.

Sin embargo, a pesar de estos y otros trabajos sobre los humedales de la ciudad, todavía no se conocen las características y las dinámicas bióticas de su flora. En este sentido, el objetivo del presente estudio fue analizar la vegetación y las coberturas de la tierra en la cuenca del humedal Jaboque y del parque La Florida considerando los siguientes aspectos: 1) composición florística, 2) estructura de la vegetación terrestre, y 3) cambios en la cobertura de la tierra entre el 2004 y el 2016.

\section{Materiales y métodos}

Área de estudio. El área de estudio se ubica a $2.600 \mathrm{msnm}$, dentro de la región denominada Sabana de Bogotá, en la zona de vida de bosque seco montano bajo (Espinal \& Montenegro, 1963). La temperatura media anual es de $13,6{ }^{\circ} \mathrm{C}$, con una humedad relativa media anual de $80 \%$, una precipitación anual de 792,8 mm, y un régimen bimodal tetraestacional de distribución de las lluvias dividido en dos periodos húmedos (de septiembre a noviembre y de marzo a abril), y dos períodos secos (de diciembre a febrero y de junio a agosto) (Hernández \& Rangel, 2009; Beltrán, 2012).

La cuenca del humedal Jaboque está situada en la localidad de Engativá, al occidente de Bogotá, donde limita al noroccidente con el río Bogotá, con el Aeropuerto Internacional El Dorado y con la Autopista Medellín (Calle 80). Aquí se encuentra el Parque Ecológico Distrital Humedal Jaboque, el segundo más grande de la ciudad con 148,5 hectáreas (ADESSA \& EAAB, 2006), el cual fue declarado como área importante para la conservación de aves de Colombia y el mundo (Hernández \& Rangel, 2009) (Figura 1). Presenta un alto grado de transformación de su cobertura vegetal y es uno de los humedales más contaminados del Distrito Capital a causa del mal manejo de vertimientos y aguas residuales (Castro, Cruz, \& Moreno, 2005; Avila \& Estupiñan, 2006; Acherman, 2007). Cuenta con un sector al norte que no se incluye oficialmente dentro de sus límites y se encuentra
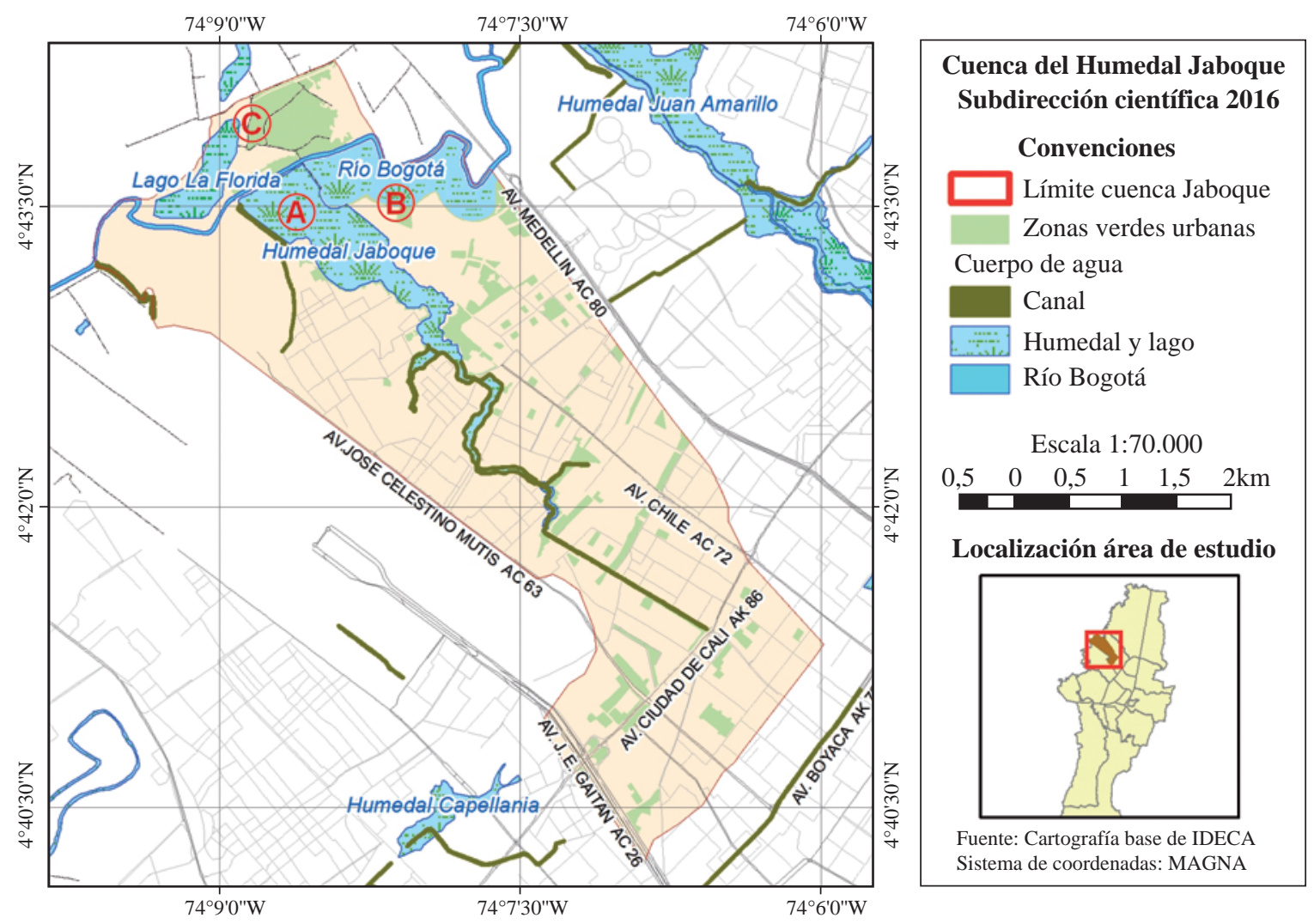

Figura 1. Mapa de la cuenca del humedal Jaboque y el parque La Florida. A) Humedal Jaboque. B) Jaboque Norte. C) Parque La Florida 
separado del cuerpo principal por una ciclovía; para efectos del presente estudio se ha denominado a esta sección “Jaboque Norte” (Figura 1). Por su parte, el Parque Regional La Florida está ubicado en el noroccidente de Bogotá, entre los municipios de Funza y Cota (Figura 1); corresponde a un área verde periurbana de carácter recreativo y deportivo con un humedal también declarado como área importante para la conservación de aves y que, por su cercanía con el río Bogotá y el humedal Jaboque, presta servicios ambientales importantes para la región (Benítez, Morales, \& CelyFajardo, 2004). La ronda de los humedales se definió en el presente trabajo como la zona no inundable incluida dentro del polígono de cada humedal en la cartografía oficial del Distrito Capital.

Riqueza y composición florística. Se hizo un muestreo en el parque La Florida y el extremo noroccidental del humedal Jaboque (Jaboque Norte) entre los meses de octubre y diciembre de 2016. El muestreo incluyó 24 parcelas de

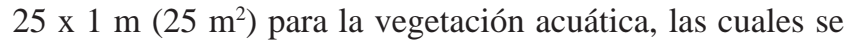
ubicaron en bandas concéntricas partiendo de la zona litoral hasta un metro de profundidad de la columna de agua. Para la vegetación leñosa terrestre se utilizaron cuatro parcelas de 50 × $2 \mathrm{~m}\left(100 \mathrm{~m}^{2}\right)$. En todas las parcelas se estimó el porcentaje de cobertura de las especies mediante el método punto-intercepto (Matteucci \& Colma, 1982), registrando el contacto de las especies en 50 puntos dentro de cada parcela. También se midió la circunferencia a la altura del pecho (CAP) y la altura de los individuos leñosos presentes con un CAP mayor de $6,25 \mathrm{~cm}$ (2 $\mathrm{cm}$ de diámetro). Se hizo el registro fotográfico de cada especie encontrada y se recolectaron muestras botánicas, las cuales se preservaron para su posterior secado e identificación en el herbario del Jardín Botánico de Bogotá (JBB). Para complementar la información florística, se incluyó la información disponible en el trabajo de Hernández \& Rangel (2009), los estudios de ADESSA \& EAAB (2006) y Córdoba (2016), así como en las colecciones de los herbarios COL y JBB. Con ayuda del programa EstimateS 9.0 (Colwell, 2013) se estimó la riqueza esperada usando los modelos Chao 1 , para los sitios en donde se hicieron parcelas, y Chao 2 para la ronda del humedal Jaboque en donde se hizo un inventario de los individuos leñosos con CAP mayor de 6,25 cm. Las plantas se clasificaron utilizando los trabajos del Angiosperm Phylogeny Group (APG, 2009) para las angiospermas, de Smith, et al. (2006) para los helechos, de Goffinet (2009) para los musgos y de Crandall-Stotler, et al. (2009) para las hepáticas.

Estructura de la vegetación terrestre. La estructura de la vegetación terrestre se analizó según los parámetros del índice de valor de importancia (IVI) de la densidad, la dominancia y la frecuencia (Rangel \& Velásquez, 1997). Se analizó la zona de la ronda del humedal Jaboque, y en el parque La Florida, aquellas zonas poco transitadas en donde era posible observar regeneración natural (margen norte del parque).
Análisis temporal de las coberturas de la tierra. Para el análisis de coberturas se adaptó la leyenda de coberturas de la tierra basada en la metodología CORINE Land Cover (IDEAM, 2010), detallada hasta el nivel cinco a una escala de 1:5.000 para su interpretación. Se definieron cuatro coberturas vegetales para las zonas inundables según su fisonomía y especies dominantes siguiendo el esquema general presentado en el protocolo de recuperación y rehabilitación ecológica de humedales en centros urbanos (Van der Hammen, et al. 2008): a) pradera errante emergente, b) pradera emergente herbácea, c) pradera emergente juncoide, y d) pradera-matorral mixto (por la mezcla de especies y fisionomías observada, la cual incluye la pradera emergente graminoide y el matorral mixto). Para el año 2004 la interpretación se generó a partir del fotomosaico 2004 de la Infraestructura de Datos Espaciales para el Distrito Capital (IDECA), y se respaldó con el estudio sobre la vegetación del humedal Jaboque realizado en el año 2003 por Hernández \& Rangel (2009). Para el año 2016, las coberturas se interpretaron a partir de una imagen de satélite SPOT del 2016 proporcionada por el Fondo Distrital para la Gestión de Riesgos y Cambio Climático (FONDIGER), del fotomosaico 2014 de la IDECA, y de la verificación de campo.

\section{Resultados}

Riqueza y composición florística. En total se encontraron 306 especies, 207 géneros y 91 familias, las cuales se presentan en la lista completa de la Tabla 1S, https://www.raccefyn.co/index. php/raccefyn/article/downloadSuppFile/496/2208. El número de especies acuáticas y terrestres fue mayor en el humedal Jaboque que en los humedales de La Florida y Jaboque Norte (Tabla 1). El grupo de plantas con mayor número de especies fue el de las eudicotiledóneas, seguido de las monocotiledóneas, con algunos representantes de monilófitos (helechos), briófitos sensu lato y algas carófitas, pero en los humedales la proporción de monocotiledóeas fue mayor (Tabla 1). Entre las familias con mayor riqueza se encontraron las siguientes: Asteraceae, Cyperaceae, Myrtaceae, Solanaceae, Fabaceae, Rosaceae, Poaceae, Polygonaceae, Malvaceae y Moraceae; en la vegetación acuática de los humedales predominaron las Asteraceae, Cyperaceae, Solanaceae, Fabaceae y Poaceae, en tanto que en la vegetación terrestre de la ronda del humedal Jaboque y el parque La Florida predominaron las Myrtaceae, Solanaceae, Fabaceae, Rosaceae y Moraceae (Tabla 2S, https:// www.raccefyn.co/index.php/raccefyn/article/download SuppFile/496/2209). Los géneros con mayor riqueza fueron los de Eleocharis, Ficus, Solanum, Juncus, Prunus, Citrus, Hydrocotyle, Persicaria, Eucalyptus, Thelypteris; en la vegetación acuática de los humedales predominaron los de Eleocharis, Solanum, Juncus, Hydrocotyle y Persicaria, en tanto que en la vegetación terrestre de la ronda del humedal Jaboque y el parque La Florida predominaron los de Ficus, Prunus, Citrus, Eucalyptus y Myrcianthes (Tabla 2S).

Según el modelo Chao 1, basado en la presencia de las especies, el muestreo registró el $79 \%$ de la riqueza total 
Tabla 1. Patrón de distribución de la riqueza según los grandes grupos taxonómicos en las diferentes zonas del área de estudio

\begin{tabular}{|c|c|c|c|c|c|}
\hline Zona de la cuenca & División & Clase & Familias & Géneros & Especies \\
\hline \multirow[t]{5}{*}{ Humedal La Florida } & Marchantiophyta & Marchantiopsida & 1 & 1 & 1 \\
\hline & Monilophyta & Polypodiopsida & 4 & 4 & 5 \\
\hline & \multirow[t]{2}{*}{ Magnoliophyta } & Eudicotyledoneae & 24 & 48 & 56 \\
\hline & & Monocotyledoneae & 7 & 12 & 16 \\
\hline & \multicolumn{2}{|l|}{ Total Humedal La Florida } & 36 & 65 & 78 \\
\hline \multirow{5}{*}{$\begin{array}{l}\text { Zona recreativa del parque } \\
\text { La Florida }\end{array}$} & Monilophyta & Polypodiopsida & 3 & 3 & 6 \\
\hline & Pinophyta & Pinopsida & 1 & 1 & 1 \\
\hline & \multirow[t]{2}{*}{ Magnoliophyta } & Eudicotyledoneae & 26 & 38 & 41 \\
\hline & & Monocotyledoneae & 5 & 6 & 6 \\
\hline & \multicolumn{2}{|l|}{ Total Zona recreativa La Florida } & 35 & 48 & 54 \\
\hline \multirow[t]{7}{*}{ Humedal Jaboque } & Charophyta & Zygnematophyceae & 1 & 1 & 1 \\
\hline & Bryophyta & Bryopsida & 2 & 3 & 3 \\
\hline & Marchantiophyta & Jungermanniopsida & 1 & 1 & 1 \\
\hline & Monilophyta & Polypodiopsida & 5 & 5 & 5 \\
\hline & \multirow[t]{2}{*}{ Magnoliophyta } & Eudicotyledoneae & 25 & 56 & 73 \\
\hline & & Monocotyledoneae & 10 & 20 & 33 \\
\hline & \multicolumn{2}{|l|}{ Total Humedal Jaboque } & 44 & 86 & 116 \\
\hline \multirow[t]{5}{*}{ Ronda del humedal Jaboque } & Pinophyta & Pinopsida & 4 & 6 & 7 \\
\hline & \multirow[t]{3}{*}{ Magnoliophyta } & Magnoliidae & 1 & 1 & 1 \\
\hline & & Eudicotyledoneae & 42 & 84 & 124 \\
\hline & & Monocotyledoneae & 4 & 8 & 10 \\
\hline & Total Ronda del humedal Jaboque & & 51 & 99 & 142 \\
\hline \multirow[t]{4}{*}{ Jaboque Norte } & Monilophyta & Polypodiopsida & 1 & 1 & 1 \\
\hline & \multirow[t]{2}{*}{ Magnoliophyta } & Eudicotyledoneae & 15 & 20 & 23 \\
\hline & & Monocotyledoneae & 6 & 12 & 16 \\
\hline & Total Jaboque Norte & & 22 & 33 & 40 \\
\hline Total general & & & 91 & 206 & 306 \\
\hline
\end{tabular}

esperada en el humedal Jaboque (Figura 2A), $90 \%$ en Jaboque Norte (Figura 2B), 76 \% en el humedal La Florida (Figura 2C) y $82 \%$ en la zona recreativa del parque La Florida (Figura 2D). Asimismo, con el modelo Chao 2 basado en la abundancia, se registró el 100 \% de la riqueza esperada en la ronda del humedal Jaboque (Figura 2E). Dado que en todas las zonas evaluadas se cruzaron los intervalos de confianza de la riqueza observada y de la esperada, se puede decir que el muestreo fue representativo (Figura 2).

Estructura de la vegetación terrestre. La vegetación terrestre del área de estudio incluyó la zona recreativa del parque La Florida y la ronda del humedal Jaboque. En la primera zona se evidenció que las especies con mayor importancia ecológica fueron Acacia melanoxylon, Pittosporum undulatum y Fraxinus chinensis. Aunque las tres presentaron una frecuencia similar, $A$. decurrens tuvo una mayor dominancia (área basal), mientras que $P$. undulatum presentó una alta densidad de individuos. Se destacó el cuarto lugar en importancia de Fuchsia boliviana, principalmente por ser relativamente frecuente y abundante, mientras que especies como Eucalyptus globulus y Quercus humboldtii sobresalieron por presentar una gran área basal (Figura 3A). Por otro lado, en la ronda del humedal Jaboque las especies con mayor importancia ecológica fueron Croton hibiscifolius, Acacia decurrens, Abatia parviflora, Prunus serotina, Schinus molle y Tecoma stans. Se destacaron C. hibiscifolius y A. decurrens por presentar las mayores áreas basales en la ronda del humedal, mientras que otras como A. parvifolia y $P$. serotina presentaron una gran densidad de individuos (más que las dos primeras especies) (Figura 3B).

Análisis temporal de las coberturas de la tierra. Se mapearon 54 coberturas en la cuenca del humedal Jaboque y el parque La Florida, 39 de ellas en el 2004 y 44 en el 2016 (Anexo, https://www.raccefyn.co/index.php/raccefyn/ article/downloadSuppFile/496/2211). En el 2004 las coberturas con mayor extensión correspondieron a territorios 


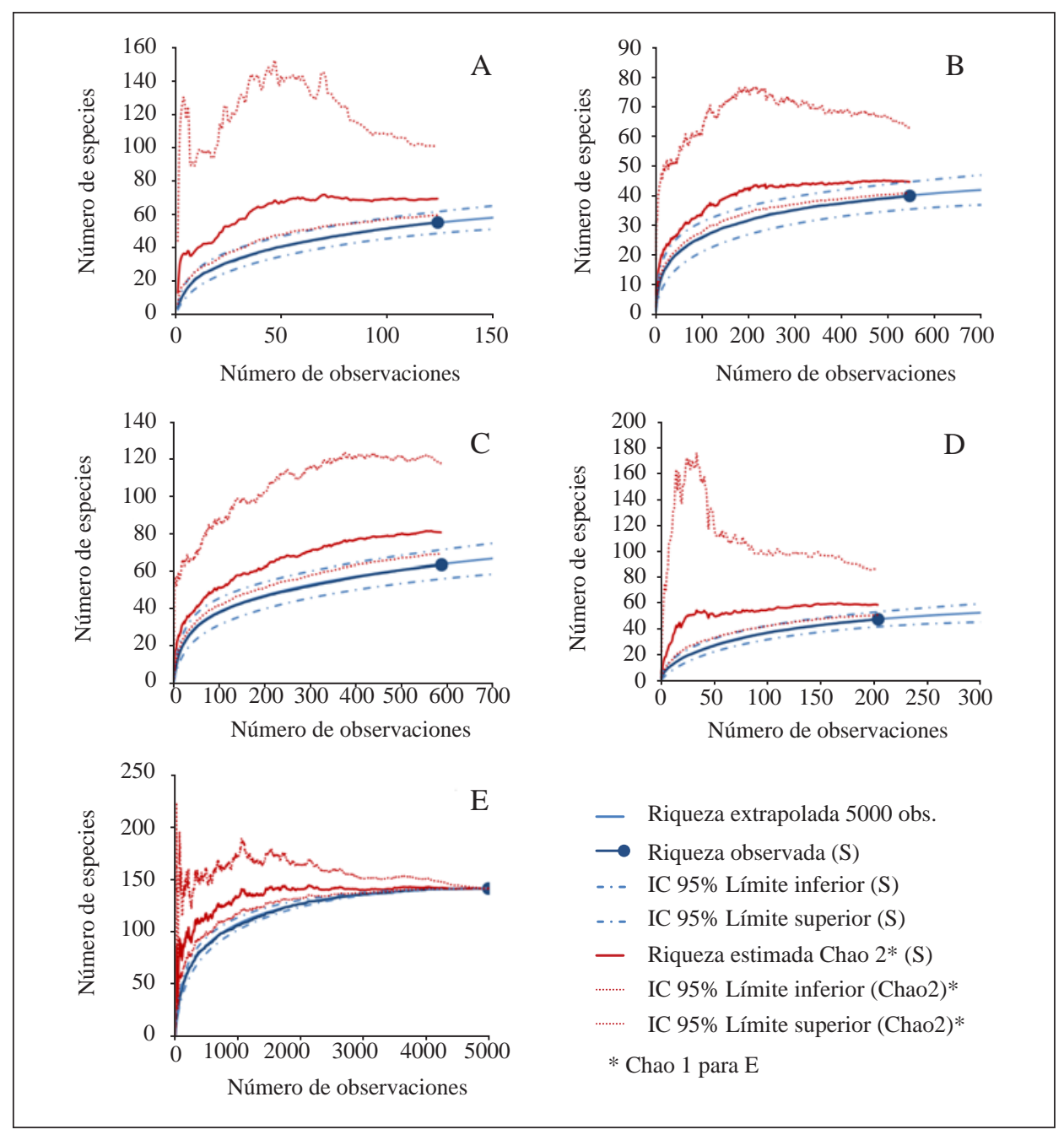

Figura 2. Curvas de acumulación de especies en la cuenca del humedal Jaboque. A) Humedal Jaboque. B) Humedal Jaboque Norte. C) Humedal La Florida. D) Zona recreativa del parque La Florida. E) Ronda del humedal Jaboque

artificializados, particularmente la cobertura denominada tejido urbano continuo (978 ha, 61 \% del área examinada) (Anexo). A esta cobertura le siguieron en extensión las coberturas de los territorios agrícolas, especialmente pastos limpios (18\%) (Figura 4). Dentro de las coberturas de bosques y áreas seminaturales, las plantaciones forestales de especies introducidas ocuparon la mayor extensión (2,7\%), y en mayor proporción, aquellas dominadas por especies de Eucalyptus (Figura 4 y Figura 5). La mayoría del tejido urbano continuo y de los pastos limpios, así como los cultivos de hortalizas, se ubicaron dentro de la cuenca del humedal Jaboque (Figura 6), mientras que la mayoría de las plantaciones forestales se encontraban en el parque La Florida (Figura 5). Asociada con las áreas húmedas, la cobertura con mayor área fue la pradera emergente juncoide (41,2 \%) (Figura 4), especialmente en donde dominaba la especie Schoenoplectus californicus (Anexo). A esta le seguían las praderas-matorrales mixtos (Figura 4), las cuales pueden estar dominadas por Ludwigia peploides, Polygonum punctatum, Bidens laevis, Lythrum maritimum, o Baccharis breviseta, entre otros (Anexo). En estas coberturas se ha reportado la presencia del helecho invasor Pteridium aquilinum, pero se trata en realidad de una identificación errónea de Hypolepis stuebelii según las muestras recolectadas en el área de estudio y en los registros del herbario COL. Las coberturas con espejo de agua (zonas pantanosas) ocuparon en este periodo menos de $4 \%$ del área de estudio y la mayoría se encontró en el humedal del parque La Florida (Figura 5). La mayor extensión de praderas emergentes juncoides se encontró en el humedal Jaboque (Figura 6 y Figura 7).

En el 2016, las coberturas que ocuparon la mayor área fueron también los territorios artificializados, en donde el tejido urbano continuo seguía en primer lugar, con un incremento de $8 \%$ con respecto al año 2004 (Anexo, Figura 6). Entre las coberturas de los territorios agrícolas, el primer lugar lo siguieron ocupando los pastos limpios $(13,4 \%)$, mientras que los cultivos de hortalizas desaparecieron completamente del humedal Jaboque a favor del 

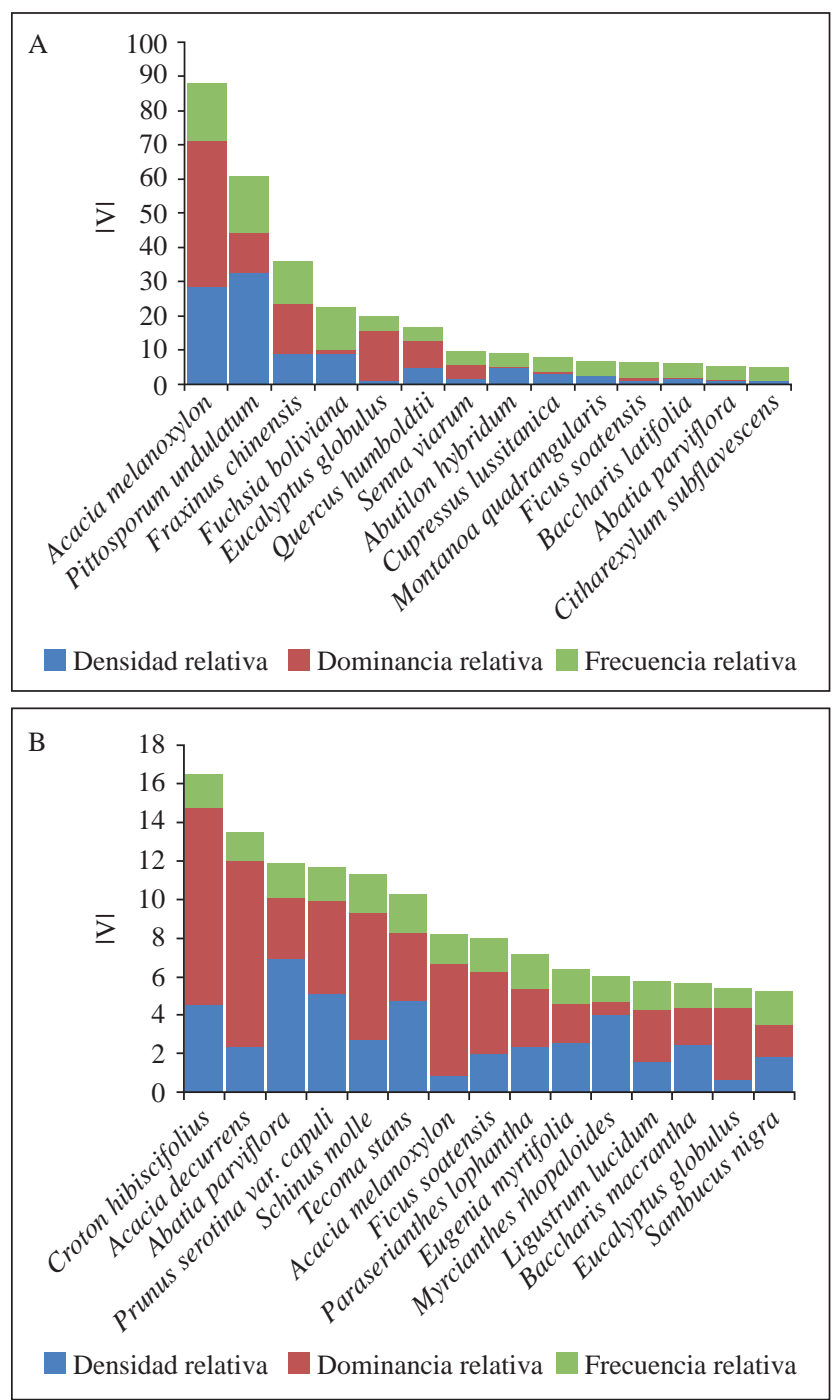

Figura 3. Estructura y biomasa de la vegetación terrestre. A) Índice de valor de importancia (IVI) en la zona recreativa del parque de La Florida. B) IVI en la ronda del humedal Jaboque

tejido urbano continuo (Figura 4, Figura 6). En cuanto a las plantaciones forestales, las de especies introducidas siguieron ocupando el primer lugar, pero habían disminuido a favor de plantaciones de especies nativas (Figura 4). En el parque La Florida las plantaciones de especies introducidas se mantuvieron en una proporción similar a la registrada en el 2004, en contraste con las que se encontraban en el humedal Jaboque Norte, aunque en este último se habían incrementado las plantaciones de especies nativas (Figura 5). En cuanto a las coberturas de áreas húmedas, se observó que la mayor extensión la ocupaban las praderas emergentes herbáceas (especialmente dominadas por B. laevis), las cuales incrementaron hasta cubrir el 43 \% de las áreas inundables, con la consecuente reducción de las praderas emergentes juncoides y las praderas-matorrales mixtos (Figura 4) dominadas por S. californicus y L. peploides, respectivamente (Anexo); los cambios más evidentes de estas últimas coberturas se produjeron en el humedal Jaboque (Figura 6 y Figura 7). Las zonas pantanosas con espejo de agua ocuparon el tercer puesto en extensión, pues casi duplicaron su área (Figura 4), especialmente en el humedal Jaboque (Figura 6 y Figura 7), y otro tanto en el humedal La Florida (Figura 5). También hubo un incremento en la cobertura de praderas errantes emergentes con respecto al 2004 (Figura 4), lo cual fue evidente en el humedal Jaboque, y sobre todo en el humedal Jaboque Norte, donde había reemplazado la cobertura de pastos limpios en muchos sectores (Figura 6 y Figura 7).

\section{Discusión}

Riqueza y composición. La riqueza de especies encontrada en los humedales del área de estudio superó la riqueza de especies acuáticas reportada para la Sabana de Bogotá (Schmidt-Mumm, 1998), especialmente en el humedal Jaboque. Una buena parte de las especies encontradas no se consideran acuáticas según las definiciones de SchmidtMumm (1998), lo cual evidencia una grave alteración de estos cuerpos de agua, que ha favorecido el avance de la vegetación terrestre y de especies exóticas como Cenchrus clandestinus. Entre las especies registradas, la mayoría pertenecen al clado de las eudicotiledóneas, un grupo cuya

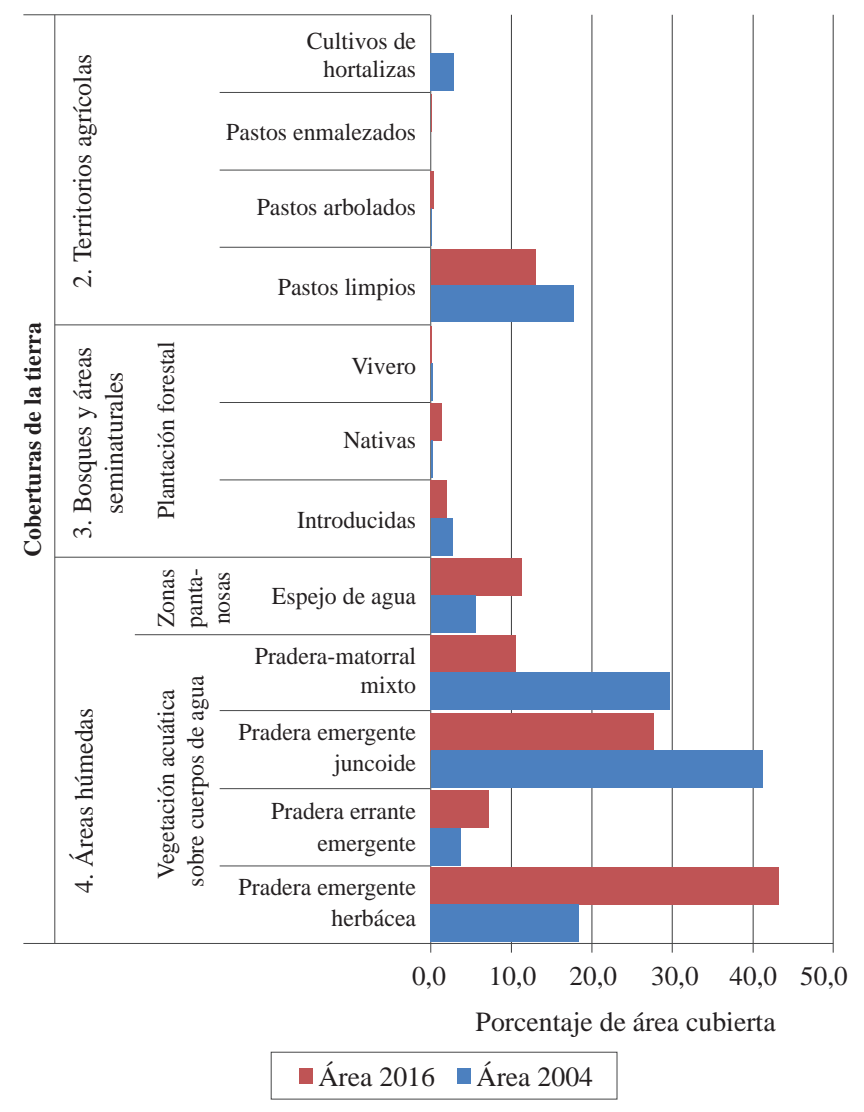

Figura 4. Porcentaje de área de las coberturas de tierra en la cuenca del humedal Jaboque y el parque La Florida en los años 2004 y 2016 excluidos los territorios artificializados y las superficies de agua 


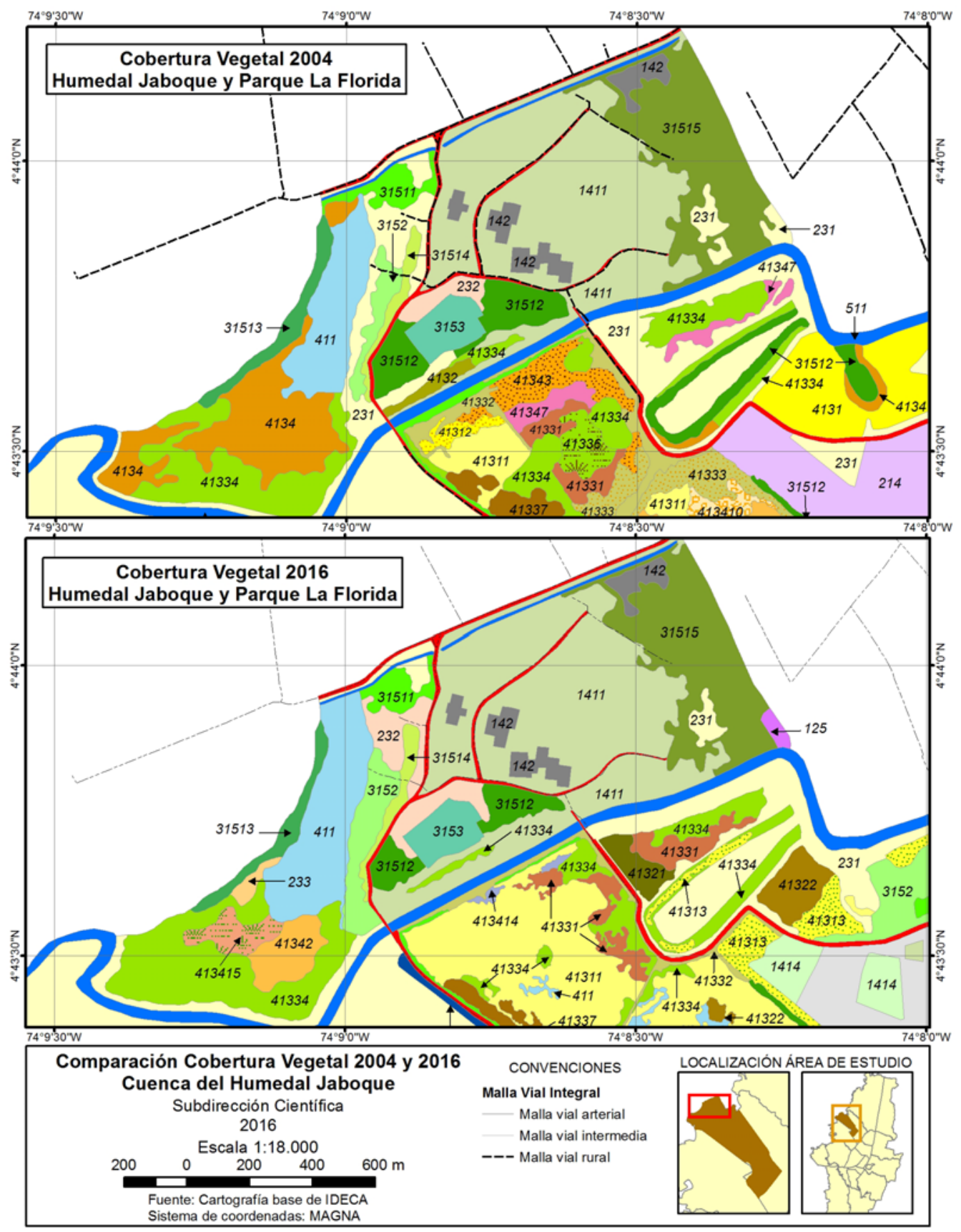

Figura 5. Mapa comparativo de coberturas vegetales en el 2004 y el 2016 en el parque La Florida. Leyenda de CORINE Land Cover de nivel 5 (ver leyenda en el Anexo)

proporción por número de especies en ambientes acuáticos se espera que corresponda a uno por uno o menos frente a las monocotiledóneas (Hutchinson, 1975). Dichas proporciones eran comunes en los humedales de la Sabana de Bogotá y el valle del río Ubaté hasta 1998 (Schmidt-Mumm, 1998), pero en los humedales evaluados las eudicotiledóneas se encontraban en una proporción de 3,5:1 en el humedal La Florida, de 2,2:1 en Jaboque y de 1,5:1 en Jaboque Norte, lo que confirma la reducción de la riqueza de plantas nativas de los humedales debido a la degradación y la pérdida de su hábitat. Paradójicamente, según este criterio el humedal que aparentemente presenta un mejor estado es Jaboque Norte, justamente el sector que no se encuentra incluido en la cartografía oficial del Distrito Capital o del Instituto Alxander von Humboldt (Escobar, 2016), y que, además, se encuentra rodeado de proyectos urbanísticos residenciales en expansión (Escobar, 2016).

En cuanto a la composición de la vegetación terrestre, se observó una proporción similar entre el número de especies nativas de la Sabana de Bogotá y de los alrededores reportado 


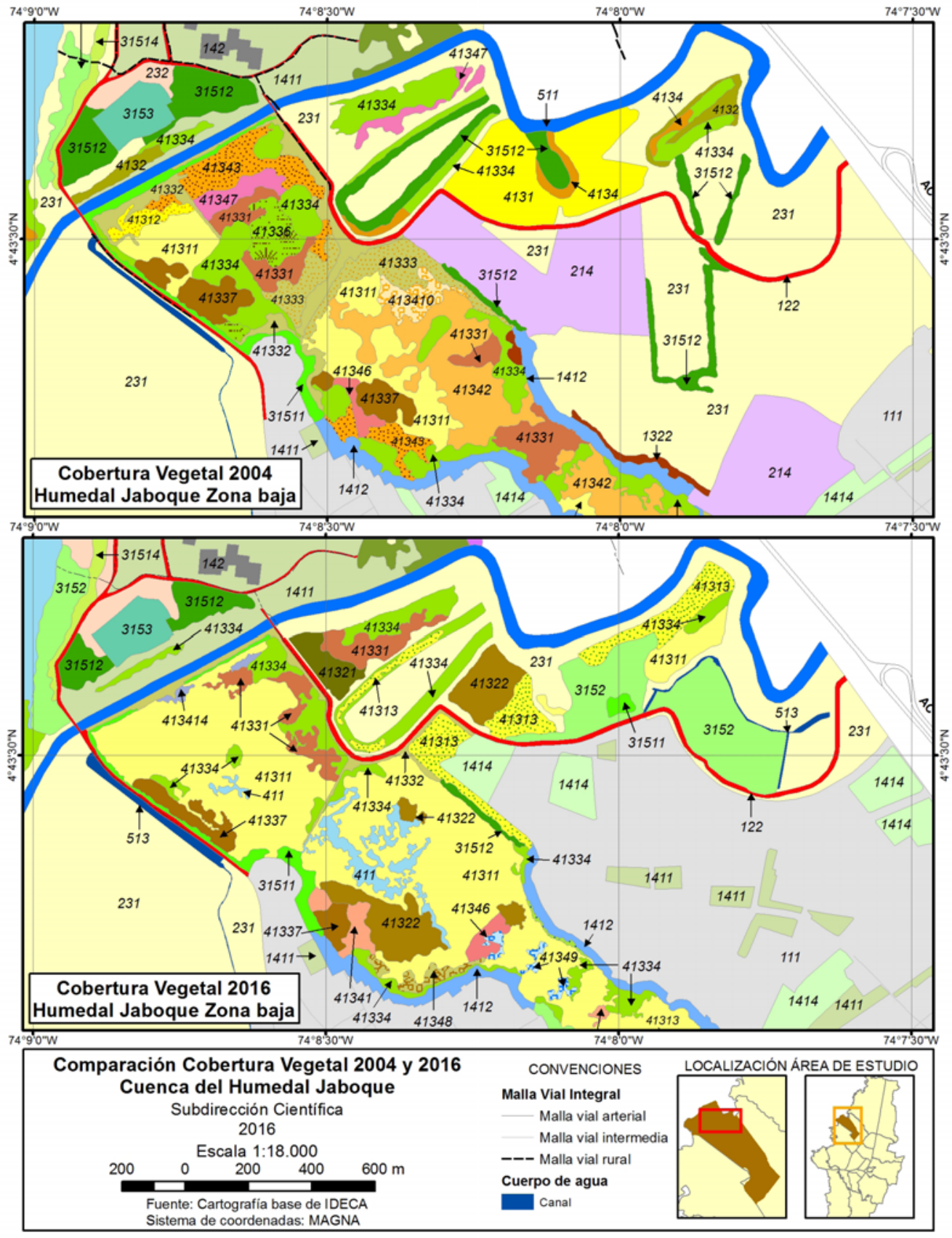

Figura 6. Mapa comparativo de coberturas vegetales en el 2004 y el 2016 en la zona baja del humedal Jaboque y Jaboque Norte. Leyenda de CORINE Land Cover, nivel 5 (ver leyenda en el Anexo)

por Cleef \& Hooghiemstra (1984), y las especies exóticas de interés ornamental y forestal (Bernal, et al., 2015), como es el caso del género Acacia. Hay que recordar que la vegetación allí presente ha sido el resultado de siembras llevadas a cabo durante las últimas décadas (ADESSA \& EAAB, 2006), de manera que la riqueza de especies nativas y exóticas no responde a un proceso natural. No obstante, el panorama no es tan desalentador si se analiza la abundancia y el área ocupada por tales especies, como se discutirá más adelante.
Estructura de la vegetación terrestre. Se destaca la importancia de especies exóticas como A. melanoxylon, $P$. undulatum, F. chinensis, A. decurrens, F. boliviana, S. molle o E. globulus, lo cual se refleja tanto en el número de individuos como en el tamaño y la frecuencia en los diferentes sectores estudiados, aunque no constituían la mayoría de las especies registradas. Como ya se mencionó anteriormente, esto se debe a las intervenciones que han realizado instituciones distritales como la Empresa de Acueducto y Alcantarillado de Bogotá o el instituto de Recreación y 


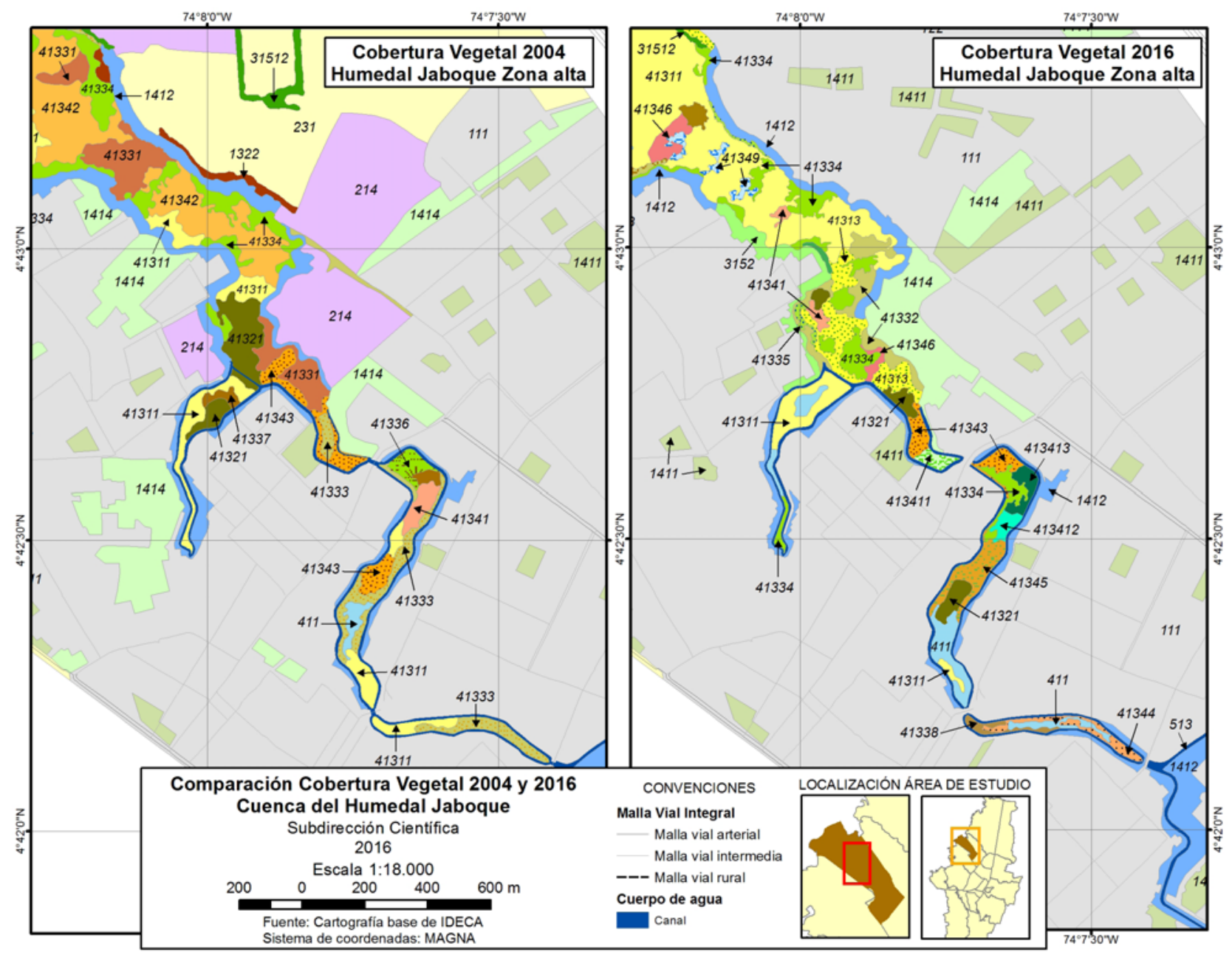

Figura 7. Mapa comparativo de coberturas vegetales en el 2004 y el 2016 en la zona alta del humedal Jaboque. Leyenda de CORINE Land Cover, nivel 5 (ver leyenda en el Anexo)

Deporte (IDRD) a través de programas de arborización de las rondas de los cuerpos de agua y de la zona recreativa de los parques (ADESSA \& EAAB, 2006; Córdoba, 2016). De cualquier manera, en el caso del parque La Florida se han introducido artificialmente una gran proporción de elementos nativos característicos de la vegetación terrestre, como Q. humboldtii, Alnus acuminata, Vallea stipularis y Salix humboldtiana, los cuales han habitado la Sabana de Bogotá desde el Pleistoceno (Cleef \& Hooghiemstra, 1984; Van der Hammen, 1986).

Análisis temporal de las coberturas de la tierra. En general, la gran abundancia y la extensión del área ocupada por especies como B. laevis, S. californicus, Juncus effusus, C. clandestinus y Eichhornia crassipes evidencian procesos de degradación del humedal Jaboque, pues estas especies son tolerantes a condiciones fisicoquímicas extremas del agua, a grandes cantidades de sedimentos, y a la contaminación por desechos domésticos, residuos sólidos, aguas residuales y materia orgánica (Castro, Cruz \& Moreno, 2005). No obstante, el aumento en el área ocupada por algunas de estas especies en los 12 años evaluados puede ser un indicador de que las condiciones físicas o bióticas han cambiado en un sentido positivo dentro de los humedales. Hay dos eventos que ilustran esta afirmación, el primero es la reducción de las coberturas dominadas por $C$. clandestinus, una especie terrestre exótica, y S. californicus, una especie helófita (enraizada en sedimento con las estructuras vegetativas sobre la superficie del agua la mayoría del tiempo) (SchmidtMumm, 1998) y, además, típica de la zona de litoral (Hernández \& Rangel, 2009); en ambos casos se redujo el área ocupada a favor de las coberturas dominadas por $B$. laevis, también helófita, pero que puede habitar a mayor profundidad en la columna de agua (Hernández \& Rangel, 2009). El segundo evento es el incremento del área ocupada por las praderas errantes emergentes, dominadas por especies pleustófitas como E. crassipes, Lemna spp o Azolla filiculoides (plantas flotantes con raíces suspendidas o sin ellas) (Schmidt-Mumm, 1998), así como el aumento del espejo de agua, todo ello en zonas donde antes había praderas emergentes con B. laevis, o incluso pastos limpios, como se 
observó en Jaboque Norte. Dado que los humedales poco profundos son susceptibles de ser colonizados por especies enraizadas emergentes o errantes (Schmidt-Mumm, 1998), la ampliación de las coberturas mencionadas podría evidenciar una mayor profundidad de los cuerpos de agua con respecto al año 2004, aunque la persistencia de B. laevis, E. crassipes y $A$. filiculoides puede indicar que la carga de nutrientes sigue siendo alta en estos humedales (SchmidtMumm, 1998; Castro, Cruz, \& Moreno, 2005; Ávila \& Estupiñan, 2006; Acherman, 2007; Beltrán \& Rangel, 2013; Rivera, Pinilla \& Rangel, 2013; Caicedo, 2016).

En este sentido, vale la pena llamar la atención sobre la necesidad de detener la urbanización en expansión que sufre el área de estudio, pues no solo implica la fragmentación, la sedimentación y la disminución de los humedales (especialmente en Jaboque Norte), sino que también provoca la eutrofización del área inundable por la recepción directa de las aguas residuales de la ciudad. En el humedal Jaboque, por ejemplo, se ha comprobado la relación directa entre el nivel de urbanización y los niveles de fósforo en el agua, pues conforme aumenta la población en las áreas circundantes aumenta igualmente la eutrofización del humedal (Caicedo, 2016). Estos niveles de contaminación tienen efectos negativos sobre la biota como la disminución de la diversidad de plantas y hábitats y del número de especies de aves, algunas de las cuales no se han vuelto a encontrar en el humedal (por ejemplo, el pato calvo Anas americana y el chorlito dorado Pluvialis dominica) (Acherman, 2007; Caicedo, 2016).

Por otro lado, si bien se ha reducido la cobertura de las plantaciones de especies exóticas en la vegetación terrestre del parque La Florida y el humedal Jaboque Norte, se impone un llamado de atención sobre las plantaciones que se han establecido dentro del área inundable en Jaboque Norte, pues podría afectar la recuperación que ha mostrado el humedal al acelerar nuevamente la colmatación y desecación debidas a la siembra de árboles.

$\mathrm{Al}$ margen de los resultados obtenidos en el presente estudio, es necesario hacer una aclaración sobre los conceptos utilizados en la definición de las plantas invasoras de los humedales de Bogotá (Díaz-Espinosa, Díaz-Triana, \& Vargas, 2012), la mayoría de las cuales correspondieron a flora acuática y palustre en el presente estudio. Esta observación se hace principalmente porque la abundancia de estas especies está relacionada con el área del litoral, la topografía y los nutrientes del agua y del suelo, de manera que proliferan naturalmente en litorales poco profundos con muchos nutrientes (Schmidt-Mumm, 1998). Teniendo esto en cuenta, la formación de parches monoespecíficos de $B$. laevis o $S$. californicus sólo es el reflejo de alteraciones más graves en la estructura y la dinámica hídrica y biótica de los humedales, producidas por acciones antrópicas (Van der Hammen, et al., 2008). Lo anterior no es coherente con un posible potencial poder invasor inherente a estas especies nativas, como se afirma en las diferentes fichas del trabajo de Díaz-Espinosa, Díaz-Triana \& Vargas (2012). El mejor ejemplo es el juncal de $S$. californicus, pues a pesar de que esta especie presenta crecimiento rápido y tiende a competir fuertemente para dominar los bordes (Díaz-Espinosa, DíazTriana \& Vargas, 2012), también provee hábitats importantes para la avifauna de los humedales, como es el caso de las monjitas (Agelaius icterocephalus) y la tingua de pico rojo (Gallinula chloropus), como se estableció en el plan de manejo del humedal Jaboque (ADESSA \& EAAB, 2006) y durante el presente estudio (observación personal). En este trabajo también se observó que el área ocupada por esta especie se había reducido en el humedal Jaboque entre los años 2004 y 2016 sin la necesidad de una erradicación artificial, y además se ha visto desplazada naturalmente por otras especies nativas más adaptadas a la vida acuática. Por último, esta especie tiene un gran potencial económico y cultural, ampliamente reconocido desde la antigüedad en los Andes de Colombia, Ecuador, Perú y Bolivia, y que sigue vigente (Macía \& Balslev, 2000; Banack, et al., 2004; Linares, et al., 2008). Por todas estas razones se debería reconsiderar el hecho de catalogar como invasoras a esta y otras especies nativas presentes en la cuenca del humedal Jaboque y el parque La Florida. Dado su potencial biótico y cultural, deberían contemplarse, por el contrario, para las estrategias de remediación y los planes de manejo y conservación a los cuales se han vinculado los residentes vecinos de estos y otros humedales de Bogotá.

\section{Conclusiones}

A pesar de que se registraron más especies en los humedales de lo que se tenía reportado en la literatura para la Sabana de Bogotá, una gran proporción de tales especies eran eudicotiledóneas terrestres, lo que evidenció la alteración de estos cuerpos de agua y el reemplazo de la flora acuática. Pese a esto, el área ocupada por coberturas de vegetación acuática era predominante en los humedales estudiados e, incluso, hubo incremento en el área de espejos de agua en el 2016, lo cual podría indicar un aumento en el nivel de agua de estos sistemas y representaría probablemente una leve recuperación con respecto a su estado en el año 2004. Aun así, algunas especies que proliferan en condiciones de aguas eutróficas, como B, laevis, E. crassipes o A. filiculoides, siguen ocupando una gran extensión. Dicho incremento de nutrientes es consecuencia directa de la acelerada expansión urbana en el área de estudio, como ha quedado documentado con el reemplazo de pastos limpios y cultivos de hortalizas presentes en el 2004 por una cobertura de tejido urbano continuo en el 2016.

Además, la vegetación terrestre estuvo representada en similar proporción por especies nativas de la Sabana de Bogotá y por especies exóticas, aunque fue evidente el predominio de estas últimas. No obstante, la cobertura de las plantaciones de especies exóticas disminuyó a favor de las nativas. Algunas nuevas especies fueron establecidas en el humedal Jaboque Norte, lo que pone en peligro la recuperación que ha presentado este cuerpo de agua desde el 2004. 


\section{Agradecimientos}

A la Subdirección Científica del Jardín Botánico de Bogotá por el apoyo logístico y la información del humedal Jaboque que fue suministrada, a los operarios Carlos Fonseca e Inelza López por el apoyo operativo en campo y en el laboratorio, y a la administración del parque La Florida por la seguridad y el acceso a zonas restringidas del parque.

\section{Información suplementaria}

Tabla 1S. Lista de plantas registradas en la microcuenca Jaboque y en el Parque La Florida. A) Humedal La Florida. B) Zona recreativa del Parque La Florida. C) Humedal Jaboque. D) Ronda del Humedal Jaboque. E) Humedal Jaboque Norte. Vea la tabla 1S en: https://www.raccefyn.co/index.php/raccefyn/article/ downloadSuppFile/496/2208

Tabla 2S. Familias y géneros con mayor riqueza de especies. A) Humedal La Florida. B) Zona recreativa del parque La Florida. C) Humedal Jaboque. D) Ronda del humedal Jaboque. E) Humedal Jaboque Norte. Vea la tabla 2S en: https://www.raccefyn.co/index. php/raccefyn/article/downloadSuppFile/496/2209

Anexo 1. Leyenda de los mapas de coberturas vegetales y comparación de cambios en área entre los años 2004 y 2016 en la cuenca del humedal Jaboque y el parque La Florida. La cifra inicial del código de la leyenda se refiere a: 1) Territorios artificializados, 2) Territorios agrícolas, 3) Bosques y áreas seminaturales, 4) Áreas húmedas, 5) Superficies de agua (ver IDEAM, 2010). Vea el anexo 1 en: https://www.raccefyn.co/index.php/raccefyn/article/ downloadSuppFile/496/2211

\section{Contribución de los autores}

DMC, toma de datos, análisis riqueza composición y estructura, escritura del manuscrito, proceso editorial. CLD, toma de datos, análisis coberturas, MYV, toma de datos. MSR, toma de datos, análisis coberturas. JWLC, toma de datos, procesamiento de muestras.

\section{Conflicto de intereses}

Los autores declaran no tener conflicto de intereses.

\section{Referencias}

Acherman, J. (2007). Análisis del estado de alteración y contaminación del humedal Jaboque. Trabajo de grado, Pontificia Universidad Javeriana, Facultad de Estudios Ambientales y Rurales, Bogotá.

ADESSA, \& EAAB. (2006). Plan de Manejo Ambiental del Humedal Jaboque. Convenio de cooperación No. 9-0724100-061-2006. Bogotá, D.C.: Asociación para el Desarrollo Social y Ambiental (ADESSA) \& Empresa de Acueducto y Alcantarillado de Bogotá (EAAB). p. 601.

Angiosperm Phylogeny Group. (2009). An update of the Angiosperm Phylogeny Group classification for the orders and families of flowering plants: APG III. Botanical Journal of the Linnean Society. 161 (2): 105-121.
Ávila, S. \& Estupiñan, S. (2006). Calidad bacteriológica del agua del humedal de Jaboque, Bogotá, Colombia. Caldasia. 28: 66-78.

Ávila, Y., Montenegro, A. \& Vargas, O. (2003). Mecanismos de persistencia de ocho especies vegetales del humedal Jaboque. Ambiente y Desarrollo. 13: 11-20.

Banack, S.A., Rondón, X. J. \& Diaz-Huamanchumo, W. (2004). Indigenous Cultivation and Conservation of Totora (Schoenoplectus Californicus, Cyperaceae) in Perú. Economic Botany. 58 (1): 11-20.

Beltrán, J. (2012). Formulación de un modelo dinámico de simulación ecológica del humedal Jaboque-Bogotá D. C., con fines de restauración y conservación. Tesis de Doctorado, Universidad Nacional de Colombia, Departamento de Biología, Bogotá.

Beltrán., J. E. \& Rangel, J.O. (2013). Modelación del estado trófico del humedal de Jaboque, Bogotá D. C., Colombia. Acta Biológica Colombiana. 18: 149-164.

Benítez, H. D., Morales, J. E. \& Cely-Fajardo, J. E. (2004). Aspectos de la reproducción y el comportamiento de Podylimbus podiceps (Aves: Podycipedidae) en dos humedales de Bogotá, Colombia. Acta Biológica Colombiana. 9: $61-68$.

Bernal, R., Gradstein, S. R. \& Celis, M. (2015). Catálogo de plantas y líquenes de Colombia. Instituto de Ciencias Naturales, Universidad Nacional de Colombia, Bogotá. Fecha de consulta: 28 de enero, 2017. Disponible en: http:// catalogoplantasdecolombia.unal.edu.co

Caicedo, A. M. (2016). Simulación dinámica del uso de suelo y los cambios en la biodiversidad de los humedales de Bogotá. Caso Jaboque, Juan Amarillo y Córdoba. Trabajo de grado, Maestría, Universidad Distrital Francisco José de Caldas, Facultad de Medio Ambiente y Recursos Naturales, Bogotá.

Castro, F., Cruz, I. \& Moreno, A. (2005). Evaluación de la calidad del agua y diagnóstico ambiental del humedal Jaboque. Tecnogestión. 2: 77-83.

Cleef, A. M. \& Hooghiemstra, H. (1984). Present vegetation of the area of the high plain of Bogotá. In H. Hooghiemstra (Ed.), Vegetation and Climatic History of the High Plain of Bogotá, Colombia. 79: 1-368. Vaduz, Liechtenstein: Ganter Verlag.

Colwell, R. K. (2013). EstimateS: Statistical estimation of species richness and shared species from samples. Versión 9. Fecha de consulta: 11 de diciembre de 2016. Disponible en: Persistent URL <purl.oclc.org/estimates>. http://viceroy. eeb.uconn.edu/estimates/

Córdoba, M. (2016). Proyecto de recuperación y restauración ecológica en el Parque Ecológico Distrital del Humedal Jaboque, con miras a la identificación e implementación de medidas de adaptación al cambio climático. Bogotá, D. C., Colombia: Convenio Interadministrativo No. 001 de 2015. Fondo Distrital para la Gestión de Riesgos y Cambio Climático (FONDIGER) \& Jardín Botánico de Bogotá José Celestino Mutis, Subdirección Técnica. p. 45.

Instituto de Hidrología, Meteorología y Estudios Ambientales IDEAM. (2010). Leyenda nacional de coberturas de la tierra. Metodología CORINE Land Cover adaptada para Colombia, escala 1: 100.000. Instituto de Hidrología Meteorología y Estudios Ambientales (IDEAM), Instituto Geográfico Agustín Codazzi (IGAC), Corporación Autónoma Regional del Magdalena (CORMAGDALENA), Bogotá, Colombia. 
Crandall-Stotler, B., Stotler, R. E. \& Long, D. G. (2009). Phylogeny and classification of the Marchantiophyta. Edinburgh Journal of Botany. 66 (1): 155-198.

Díaz-Espinosa, A. Díaz-Triana, J. \& Vargas, O. (2012). Catálogo de plantas invasoras de los humedales de Bogotá. Bogotá, Colombia: Grupo de Restauración Ecológica de la Universidad Nacional de Colombia y Secretaría Distrital de Ambiente.

Escobar, J. 2016. Naturaleza identificada: construcción colectiva de un mapa de humedales en Bogotá. En: Mejía M. A. (ed.). Naturaleza Urbana. Instituto de Investigación de Recursos biológicos Alexander von Humboldt. Bogotá D. C., Colombia. p. 208.

Espinal, L. \& Montenegro, E. (1963). Mapa de formaciones vegetales de Colombia. Bogotá D.C., Colombia: IGAC.

Goffinet, B., Buck, W. R., \& Shaw. (2009). Morphology, anatomy, and classification of the Bryophyta. En B. Goffinet \& A. J. Shaw (Eds.), Bryophyte biology (pp. 55-138).

Hernández, J. \& Rangel-Ch., J. (2009). La vegetación del humedal de Jaboque (Bogotá, D.C.). Caldasia. 31: 355-379.

Hutchinson, G. E. (1975). A treatise on limnology. Volume III. Limnological botany. Wiley.

Linares, E. L., Galeano, G., García, N. \& Figueroa, Y. (2008). Fibras vegetales empleadas en artesanías en Colombia. Bogotá: Artesanías de Colombia, Ministerio de Comercio, Industria y Turismo, Instituto de Ciencias Naturales, Facultad de Ciencias - Universidad Nacional de Colombia - Sede Bogotá.

Macía, M. J., \& Balslev, H. (2000). Use and management of totora (Schoenoplectus californicus, Cyperaceae) in Ecuador. Economic Botany. 54 (1): 82-89.
Matteucci, S. \& Colma, A. (1982). Metodología para el estudio de la vegetación. Washington D.C., USA.: Secretaría General de la Organización de Estados Americanos (OEA).

Montenegro, A., Parra, Y., Mendivelso, H., Vargas, O. (2006). Potencial del banco de semillas en la regeneración de la vegetación del humedal Jaboque, Bogotá, Colombia. Caldasia. 28: 285-306.

Rangel, J. O., \& Velásquez, A. (1997). Métodos de estudio de la vegetación. En J. Rangel, P.D., Lowy, C., \& M. Aguilar, P. (Eds.), Colombia diversidad biótica II, tipos de vegetación en Colombia. Bogotá: Instituto de Ciencias Naturales, Universidad Nacional de Colombia. p. 59-87.

Rivera, J., Pinilla-A., G. \& Rangel-Ch., J. (2013). Ensamble de macroinvertebrados acuáticos y su relación con las variables físicas y químicas en el humedal Jaboque-Colombia. Caldasia. 35: 389-408.

Schmidt-Mumm, U. (1998). Vegetación Acuática y Palustre de la Sabana de Bogotá y Plano del Río Ubaté (Tesis de Maestría). Universidad Nacional de Colombia, Departamento de Biología.

Smith, A. R., Pryer, K. M., Schuettpelz, E., Korall, P., Schneider, H., Wolf, P. G. (2006). A classification for extant ferns. Taxon. 55 (3): 705-731.

Van der Hammen, T. (1986). La Sabana de Bogotá y su lago en el Pleniglacial Medio. Caldasia. 15: 249-262.

Van der Hammen, T., Stiles, F., Roselli, L., Chisacá, M., Camargo, G., Guillot, G., Rivera, D. (2008). Protocolo de recuperación y rehabilitación ecológica de humedales en centros urbanos. Secretaría Distrital de Ambiente. Bogotá. 